\title{
The Hydrogen-bond Basicity of Carbenes
}

\author{
Michael H. Abraham, ${ }^{1}$ José Elguero, ${ }^{2}$ Ibon Alkorta ${ }^{2, *}$
}

\footnotetext{
${ }_{1}^{1}$ Department of Chemistry, University College London, 20 Gordon Street, London WC1H OAJ, UK

2 Instituto de Química Médica, CSIC, Juan de la Cierva, 3, E-28006 Madrid, Spain

* Corresponding author's e-mail address: ibon@iqm.csic.es
}

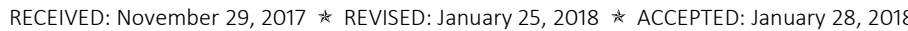

\begin{abstract}
We have evaluated theoretically in the gas-phase (MP2/aug-cc-pVTZ) the hydrogen-bond basicity of simple carbenes and vinylidenes and compared them to the corresponding nitrogen and oxygen derivatives using HF as Lewis acid. These values fit conveniently with $B$ values only if $\mathrm{sp}^{3}$ and $\mathrm{sp}^{2}$ atoms are treated separately, which is a consequence of the gas-phase (calculated) vs. solution (measured) effects.
\end{abstract}

Keywords: carbenes, N-heterocyclic carbenes, Gas-phase MP2 calculations, hydrogen-bond basicity (HBB), $\beta$ scale of HBB.

\section{INTRODUCTION}

$S$ INGLET carbenes, being Lewis-bases (LBs), form hydrogen bonds (HBs) with Lewis acids (LAs). ${ }^{[1]}$ These HBs were first studied theoretically by Pople et al. (LA = HF). ${ }^{[2,3]}$ We started our interest in these systems in 1996, ${ }^{[4]}$ and continued in 2017.[5] Several other papers were published on carbenes HBs by Standard, ${ }^{[6,7]}$ Howard, ${ }^{[8]}$ Hollóczki ${ }^{[9]}$ and Sander. ${ }^{[10]}$ However, the only data of the hydrogen bond basicity, HBB, of singlet carbenes in a scale of $\mathrm{HBB}$ is the prediction by Platts based on single point B3LYP/6-311G $(\mathrm{d}, \mathrm{p})$ calculations concerning the $B$ value of Arduengo's carbene (1,3,4,5-tetramethyl-imidazolidene) to be $0.83 . .^{[11]}$ In the Abraham scale, a value of 0.83 corresponds to a very basic substance (triethylamine, $B=$ 0.79).[12,13]

The domains where the HBB of carbenes are relevant is very large; ${ }^{[14-19]}$ as a particular interesting example we note that it has been reported that a stable carbene is present at the active site of a thiamin enzyme where weak interactions, like HBs, play a major role. ${ }^{[20]}$

\section{COMPUTATIONAL DETAILS}

All electronic structure calculations were performed with the Gaussian 09 program package. ${ }^{[21]}$ The geometries of monomers and complexes were optimized without any restriction using the second-order Møller-Plesset perturbation theory (MP2) ${ }^{[22]}$ with the aug-cc-pVTZ basis set. ${ }^{[23,24]}$ All stationary points were confirmed as minimum energy by the absence of imaginary frequencies. $E_{\mathrm{b}}$, the binding energy, is defined as the difference between the total energy of the complex and the sum of that of the isolated monomers in their minima configuration. The minima of the electrostatic potential (MEP) were analyzed with the DAMQT 2.1.0. program. ${ }^{[25]}$

\section{RESULTS AND DISCUSSION}

The aim of the present paper is to explore theoretically the $\mathrm{HBB}$ of carbenes by calculating the gas-phase binding energies with HF of a series of oxygen, nitrogen and carbon derivatives, all of them having lone pairs (LPs) some of them (oxygen and nitrogen derivatives) having known values of $B$. We have considered $s p^{3}$ carbenes (RR'C:) and $s p^{2}$ vinylidenes ( $R R^{\prime} C=C:$ ). All the studied carbenes except methylene $\left(: \mathrm{CH}_{2}\right)$ have the singlets more stable than the triplets. ${ }^{[26]}$ The same happens with the vinylidenes (this work) where the singlet/triplet gaps are $-218.6 \mathrm{~kJ} \cdot \mathrm{mol}^{-1}$ (: $\left.\mathrm{CCH}_{2}\right)$ and $-80.3 \mathrm{~kJ} \cdot \mathrm{mol}^{-1}\left(: \mathrm{CCF}_{2}\right)$.

In a first attempt we selected the compounds represented in Scheme 1.

But, during the optimization process of both carbenes and vinylidenes, if $R, R^{\prime}, R^{\prime \prime}$ is $\mathrm{CH}_{3}$, then a 1,2hydrogen shift ${ }^{[27]}$ occurs and the optimized structure is an olefin. In two vinylidenes, $\left(\mathrm{CH}_{3}\right)_{2} \mathrm{C}=\mathrm{C}$ : and $\left(\mathrm{NH}_{2}\right)_{2} \mathrm{C}=\mathrm{C}$ :, the 
Table 1. Calculated (gas-phase) and experimental (solution) measures of HBB: $\beta_{2}^{H}$, and $B$ values are all from Abraham ${ }^{[12,13,18]}$ except one value (imidazolidene) from Platts. ${ }^{[11]}$

\begin{tabular}{|c|c|c|c|c|c|}
\hline Bases & $E_{\mathrm{b}} / \mathrm{kJ} \cdot \mathrm{mol}^{-1}$ & Abraham $B$ & Predicted $B$ & Abraham $\beta_{2}^{H(b)}$ & MEP-min / kJ. $\mathrm{mol}^{-1}$ \\
\hline \multicolumn{6}{|l|}{$\mathrm{sp}^{3}$} \\
\hline $\mathrm{NH}_{3}$ (ammonia) & -54.1 & 0.56 & & 0.60 & \\
\hline $\mathrm{CH}_{3} \mathrm{NH}_{2}$ (methylamine) & -60.1 & 0.58 & & 0.70 & \\
\hline$\left(\mathrm{CH}_{3}\right)_{2} \mathrm{NH}$ (dimethylamine) & -63.0 & 0.66 & & 0.72 & \\
\hline $\mathrm{H}_{2} \mathrm{O}$ (water) & -37.5 & 0.46 & & 0.38 & \\
\hline $\mathrm{CH}_{3} \mathrm{OH}$ (methanol) & -42.9 & 0.47 & & 0.41 & \\
\hline$\left(\mathrm{CH}_{3}\right)_{2} \mathrm{O}$ (dimethyl ether) & -45.6 & 0.41 & & 0.43 & \\
\hline$: \mathrm{CH}_{2}$ (methylene) & -49.3 & & 0.51 & & -241.5 \\
\hline$: \mathrm{C}\left(\mathrm{CH}_{3}\right)_{2}$ & -68.1 & & 0.68 & & -306.4 \\
\hline$: \mathrm{CH}\left(\mathrm{OCH}_{3}\right)$ & -64.4 & & 0.65 & & -280.9 \\
\hline$: \mathrm{CH}\left(\mathrm{NH}_{2}\right)$ & -71.3 & & 0.71 & & -328.2 \\
\hline$: \mathrm{C}\left(\mathrm{NH}_{2}\right)_{2}$ & -73.0 & & 0.72 & & -330.8 \\
\hline Arduengo's carbene (imidazolidene) & -83.6 & $0.83^{(\mathrm{a})}$ & & & -359.7 \\
\hline \multicolumn{6}{|l|}{$\mathrm{sp}^{2}$} \\
\hline $\mathrm{H}_{2} \mathrm{C}=\mathrm{NH}$ & -52.0 & & 0.63 & & \\
\hline $\mathrm{CH}\left(\mathrm{CH}_{3}\right)=\mathrm{NH}$ & -61.0 & & 0.81 & & \\
\hline$\left(\mathrm{CH}_{3}\right)_{2} \mathrm{C}=\mathrm{NH}$ & -57.1 & & 0.73 & & \\
\hline $\mathrm{CH}\left(\mathrm{CH}_{3} \mathrm{O}\right)=\mathrm{NH}$ (methyl formimidate) & -55.4 & & 0.70 & & \\
\hline $\mathrm{CH}\left(\mathrm{NH}_{2}\right)=\mathrm{NH}$ (formamidine) & -77.6 & & 1.14 & & \\
\hline $\mathrm{C}\left(\mathrm{NH}_{2}\right)_{2}=\mathrm{NH}$ (guanidine) & -71.3 & 1.04 & & & \\
\hline $\mathrm{F}_{2} \mathrm{C}=\mathrm{NH}$ & -37.1 & & 0.34 & & \\
\hline $\mathrm{H}_{2} \mathrm{C}=\mathrm{O}$ (formaldehyde) & -35.8 & 0.33 & & & \\
\hline $\mathrm{CH}\left(\mathrm{CH}_{3}\right)=\mathrm{O}$ (acetaldehyde) & -41.7 & 0.45 & & 0.38 & \\
\hline$\left(\mathrm{CH}_{3}\right)_{2} \mathrm{C}=\mathrm{O}$ (acetone) & -45.8 & 0.49 & & 0.49 & \\
\hline $\mathrm{CH}\left(\mathrm{CH}_{3} \mathrm{O}\right)=\mathrm{O}$ (methyl formate) & -39.0 & 0.38 & & 0.39 & \\
\hline $\mathrm{CH}\left(\mathrm{NH}_{2}\right)=\mathrm{O}$ (formamide) & -56.1 & 0.60 & & 0.61 & \\
\hline $\mathrm{C}\left(\mathrm{NH}_{2}\right)_{2}=\mathrm{O}$ (urea) & -59.3 & 0.84 & & & \\
\hline $\mathrm{F}_{2} \mathrm{C}=\mathrm{O}$ & -20.1 & & 0.00 & & \\
\hline $\mathrm{H}_{2} \mathrm{C}=\mathrm{C}$ : (vinylidene) & -37.7 & & 0.35 & & -189.0 \\
\hline $\mathrm{F}_{2} \mathrm{C}=\mathrm{C}$ : (difluorovinylidene) & -28.3 & & 0.16 & & -128.6 \\
\hline
\end{tabular}

(a) Platts value. ${ }^{[11]}$

(b) $\beta_{2}^{H}$ values fit slightly better but there are less compounds.

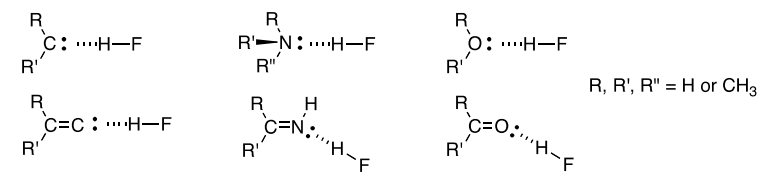

Scheme 1.

optimization leads to structures where the terminal carbon approaches the substituent $\left(\mathrm{CH}_{3}\right.$ or $\left.\mathrm{NH}_{2}\right)$. Thus, we calculated another series of compounds (Figure 1 and Table 1 ).

We have reported the values in Table 1. We give two different scales of HBB. The $\beta_{2}^{H}$ scale of Abraham et al. ${ }^{[18]}$ denotes the solute HBB towards a single hydrogen bond acid and the $B$-scale of Abraham et al. ${ }^{[11,12]}$ denotes the solute HBB when surrounded by hydrogen bond acids.

We have represented the $B$ values against the $E_{\mathrm{b}}$ ones and it is apparent that the $\mathrm{sp}^{3}$ and $\mathrm{sp}^{2}$ atoms belong to two different families (Figure 2). The use of $\Delta H$ instead of $E_{\mathrm{b}}$ leads to almost identical results (see Supporting Information).

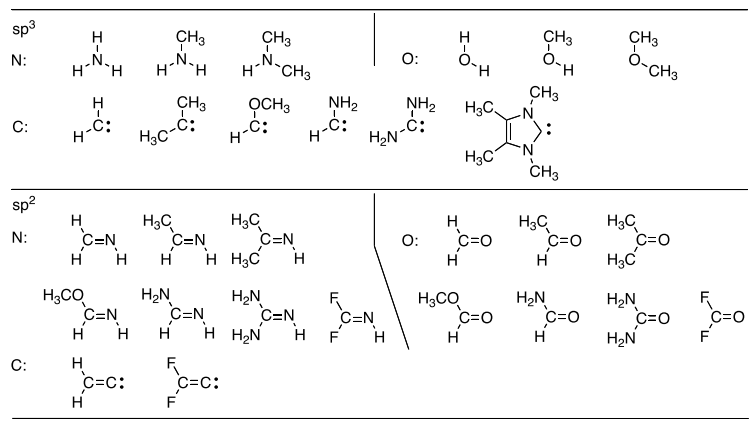

Figure 1. Compounds studied in this work.

The corresponding regression lines are:

$B=(0.08 \pm 0.07)-(0.009 \pm 0.001) E_{\mathrm{b}} \mathrm{sp}^{3}, n=7, R^{2}=0.924$, $\mathrm{RMS}$ residual $=0.04$

$B=-(0.4 \pm 0.1)-(0.020 \pm 0.002) E_{\mathrm{b}} \mathrm{sp}^{2}, n=7, R^{2}=0.954$, $\mathrm{RMS}$ residual $=0.06$ 


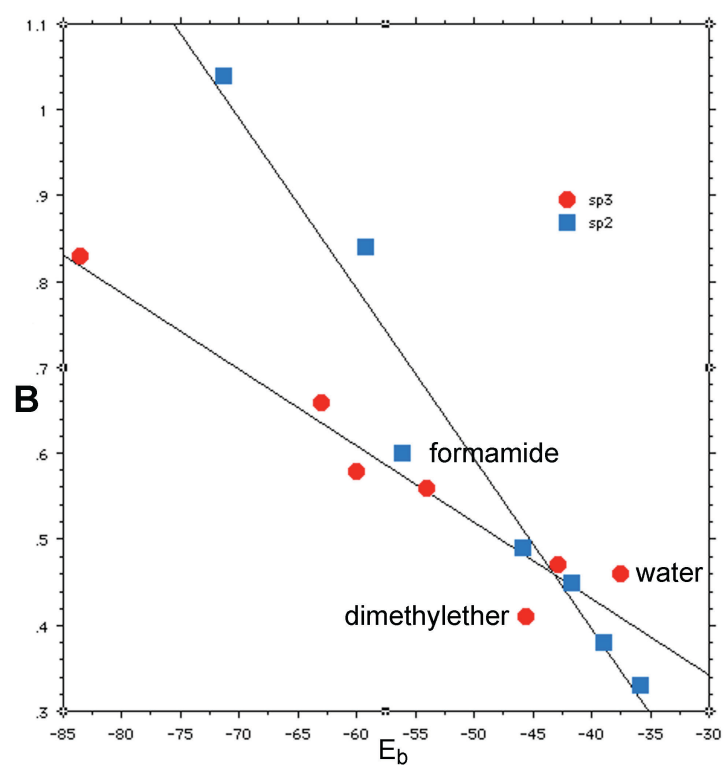

Figure 2. Scattergram of $B v s$. $E_{\mathrm{b}}$. Red dots, $\mathrm{sp}^{3}$ atoms, blue squares, $\mathrm{sp}^{2}$ atoms. The three out-of-line points are labeled.

The first equation includes Arduengo's carbene (measure 0.83, fitted 0.82). Using these equations we have predicted a number of $B$ values as shown in Table 1.

The fact that $\mathrm{sp}^{2}$ and $\mathrm{sp}^{3}$ basic centers are different is probably due to the fact that the calculated $E_{\mathrm{b}}$ values correspond to the gas-phase while the $B$ values have been obtained from solution data. The fact that gas-phase and solution greatly affect basicity is well known. ${ }^{[28]}$

We have calculated the minima of the electrostatic potential (MEP) of the eight singlet carbenes in Table 1 to see if there is a correlation with $E_{\mathrm{b}}{ }^{[11,29-32]}$ Effectively both magnitudes are related: $E_{\mathrm{b}}=(5.2 \pm 3.5)+(0.24 \pm 0.01) \mathrm{MEP}, n$ $=8, R^{2}=0.984, \mathrm{RMS}$ Residual $=2.6 \mathrm{~kJ} \cdot \mathrm{mol}^{-1}$.

\section{CONCLUSION}

Coming back to our initial question about the HBB of carbenes, we have summarized in Figure 3 the results we have obtained.

Figure 3 shows that the most frequent order of hydrogen-bond basicity is $\mathrm{N}>\mathrm{C}>\mathrm{O}$ but that there is a case of $\mathrm{C}>\mathrm{N}>0 . \mathrm{O}^{[11]}$ Thermodynamic carbon basicity (protonation) is illustrated in a paper about carbon superbases. ${ }^{[33]}$ In conclusion, carbenes and vinylidenes are, in general, less basic than nitrogen derivatives and, always, more basic than oxygen derivatives, if the structures are similar. On the other hand, hybridization and substituents play an important role on the HBB.

Acknowledgment. This work was carried out with financial support from the Ministerio de Economía, Industria y

\begin{tabular}{|c|c|c|c|c|c|c|}
\hline $\mathrm{sp}^{3}$ & $\begin{array}{c}\mathrm{H}_{-54.1}^{\mathrm{H}_{1}^{\prime}} \\
-54\end{array}$ & $\begin{array}{c}\stackrel{H}{1} \\
\mathrm{H}^{-C}: \\
-49.3\end{array}$ & 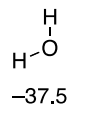 & $\begin{array}{c}\mathrm{CH}_{3} \\
\stackrel{\mathrm{I}}{\mathrm{C}:} \\
\mathrm{H}_{3} \mathrm{C}^{-}{ }^{-68.1} \\
-{ }^{2} .\end{array}$ & $\begin{array}{c}{\stackrel{\mathrm{C}}{\mathrm{i}} \mathrm{H}_{3}}_{\mathrm{H}^{-}}^{\mathrm{N}_{-}} \mathrm{CH}_{3} \\
-63.0\end{array}$ & $\begin{array}{l}\mathrm{CH}_{3} \\
\text { O- } \mathrm{CH}_{3} \\
{ }_{-45.6}\end{array}$ \\
\hline $\mathrm{sp}^{2}$ & $\begin{array}{l}\mathrm{H}_{\mathrm{C}}^{\mathrm{H}}=\mathrm{N} \\
\mathrm{H}^{\mathrm{H}} \\
-52.0\end{array}$ & $\begin{array}{l}{ }_{\mathrm{H}^{\prime}}^{\mathrm{H}}=\mathrm{C} \\
-37.7\end{array}$ & $\begin{array}{l}{ }_{C}^{H}=0 \\
H^{\prime}=0 \\
-35.8\end{array}$ & $\begin{array}{l}\mathrm{F}_{\mathrm{C}} \\
\mathrm{F}^{\mathrm{C}=\mathrm{N}} \\
-37.1\end{array}$ & $\begin{array}{l}\mathrm{F}_{\mathrm{C}}^{\mathrm{C}}=\mathrm{C}: \\
\mathrm{F}^{\prime} \\
-28.3\end{array}$ & $\begin{array}{c}\underset{\mathrm{C}}{\mathrm{F}}= \\
\mathrm{F}^{\prime} \\
-20.1\end{array}$ \\
\hline
\end{tabular}

$\mathrm{N}>\mathrm{C}>\mathrm{O}, \mathrm{C}>\mathrm{N}>\mathrm{O}, \mathrm{N}>\mathrm{C}>\mathrm{O}, \mathrm{N}>\mathrm{C}>\mathrm{O}$

Figure 3. Orders of hydrogen-bond basicity.

Competitividad (Project No. CTQ2015-63997-C2-2-P) and Comunidad Autónoma de Madrid (Project FOTOCARBON, ref S2013/MIT-2841).

Supplementary Information. Atomic coordinates of all molecules in their optimized structures. This material is available free of charge, attached to the electronic version of the article at: http://doi.org/10.5562/cca3258.

\section{REFERENCES}

[1] A. M. O'Donoghue, R. S. Massey, Acid-Base Chemistry of Carbenes, in Contemporary Carbene Chemistry (Eds.: R. A. Moss, M. P. Doyle), Wiley, Hoboken, New Jersey, 2014.

[2] J. A. Pople, K. Raghavachari, M. J. Frisch, J. S. Binkley, P. v. R. Schleyer, J. Am. Chem. Soc. 1983, 105, 6389.

[3] J. A. Pople, Chem. Phys. Lett. 1986, 132, 144.

[4] I. Alkorta, J. Elguero, J. Phys. Chem. 1996, 100, 19367.

[5] J. E. Del Bene, I. Alkorta, J. Elguero, Chem. Phys. Lett. 2017, 675, 46.

[6] L. L. Zub, J. M. Standard, J. Mol. Struct. (Theochem) 1996, 368, 133.

[7] J. M. Standard, J. Phys. Chem. A 2017, 121, 381.

[8] S. T. Howard, C. D. Abernethy, J. Comput. Chem. 2004, 25, 649.

[9] O. Hollóczki, Phys. Chem. Chem. Phys. 2016, 18, 126.

[10] G. Richter, E. Mendez-Vega, W. Sander, J. Phys. Chem. A 2016, 120, 3524.

[11] J. A. Platts, Phys. Chem. Chem. Phys. 2000, 2, 3115.

[12] M. H. Abraham, Chem. Soc. Rev. 1993, 22, 73.

[13] UFZ-LSER database [Internet]. Helmholtz Centre for environmental Research-UFZ, Leipzig, Germany.

[14] G. Bertrand, Carbene Chemistry, Marcel Dekker, New York, 2002.

[15] O. Kühl, Functionalized N-Heterocyclic Carbene Complexes, Wiley, Chichester, 2010.

[16] S. P. Nolan, N-Heterocyclic Carbenes in Synthesis, Wiley-VCH, Weinheim, 2006.

[17] C. S. J. Cazin, N-Heterocyclic Carbenes in Transition Metal Catalysis and Organocatalysis, Springer, Dordrech, 2011. 
[18] S. Diez-Gonzalez, N-Heterocyclic Carbenes: From Laboratory Curiosities to Efficient Synthetic Tools, 2nd Edition, Royal Society of Chemistry, Cambridge, 2016.

[19] H. V. Huynh, The Organometallic Chemistry of $\mathrm{N}$ Heterocyclic Carbenes, Wiley, Chichester, 2017.

[20] D. Meyer, P. Neumann, R. Ficner, K. Tittmann, Nature Chem. Biol. 2013, 9, 488.

[21] Gaussian 09, Revision E.01, M. J. Frisch, G. W. Trucks, H. B. Schlegel, G. E. Scuseria, M. A. Robb, J. R. Cheeseman, G. Scalmani, V. Barone, B. Mennucci, G. A. Petersson, H. Nakatsuji, M. Caricato, X. Li, H. P. Hratchian, A. F. Izmaylov, J. Bloino, G. Zheng, J. L. Sonnenberg, M. Hada, M. Ehara, K. Toyota, R. Fukuda, J. Hasegawa, M. Ishida, T. Nakajima, Y. Honda, O. Kitao, H. Nakai, T. Vreven, J. A. Montgomery, Jr., J. E. Peralta, F. Ogliaro, M. Bearpark, J. J. Heyd, E. Brothers, K. N. Kudin, V. N. Staroverov, R. Kobayashi, J. Normand, K. Raghavachari, A. Rendell, J. C. Burant, S. S. Iyengar, J. Tomasi, M. Cossi, N. Rega, J. M. Millam, M. Klene, J. E. Knox, J. B. Cross, V. Bakken, C. Adamo, J. Jaramillo, R. Gomperts, R. E. Stratmann, O. Yazyev, A. J. Austin, R. Cammi, C. Pomelli, J. W. Ochterski, R. L. Martin, K. Morokuma, V. G. Zakrzewski, G. A. Voth, P. Salvador, J. J. Dannenberg, S. Dapprich, A. D. Daniels, Ö. Farkas, J. B. Foresman, J. V. Ortiz, J. Cioslowski, and D. J. Fox, Gaussian, Inc., Wallingford CT, 2009.
[22] C. Møller, M. S. Plesset, Phys. Rev. 1934, 46, 618.

[23] T. H. Dunning, J. Chem. Phys. 1989, 90, 1007.

[24] D. E. Woon, T. H. Dunning, J. Chem. Phys. 1995, 103, 45.

[25] A. Kumar, S. D. Yeole, S. R. Gadre, R. López, J. F. Rico, G. Ramírez, I. Ema, D. Zorrilla, J. Comput. Chem. 2015, 36, 2350.

[26] I. Alkorta, J. Elguero, Chem. Phys. Lett. 2018, 691, 33.

[27] D. Gerbig, P. R. Schreiner, Tunneling in the Reactions of Carbenes and Oxacarbenes, in R. A. Moss, M. P. Doyle, Contemporary Carbene Chemistry, Chapter 7, Wiley, Hoboken, New Jersey, 2014.

[28] M. H. Abraham, P. L. Grellier, D. V. Prior, J. J. Morris, P. J. Taylor, J. Chem. Soc. Perkin Trans. 2 1990, 521.

[29] J. S. Murray, P. Politzer, J. Chem. Res. S 1992, 110.

[30] I. Alkorta, I. Rozas, J. Elguero, Ber. Bunsen-Ges. Phys. Chem. 1998, 102, 429.

[31] A. A. Oliferenko, P. V. Oliferenko, J. G. Huddleston, R. D. Rogers, V. A. Palyulin, N. S. Zefirov, A. R. Katritzky, J. Chem. Inf. Comput. Sci. 2004, 44, 1042.

[32] I. Alkorta, K. Zborowski, J. Elguero, M. Solimannejad, J. Phys. Chem. A 2006, 110, 10279.

[33] J. F. Kögel, D. Margetić, X. Xie, L. H. Finger, J. Sundermeyer, Angew. Chem. Int. Ed. 2017, 56, 3090. 